PSFC/JA-11-5

\author{
Fluctuation Driven Transport and Stationary Profiles \\ J. Kesner, D.T. Garnier*, M.E. Mauel* \\ * Dept. of Applied Physics and Applied Mathematics, Columbia University
}

March 2011

\begin{abstract}
Plasma Science and Fusion Center Massachusetts Institute of Technology

Cambridge MA 02139 USA
\end{abstract}

This work was supported by the U.S. Department of Energy, Grant No. DE-FG02-

98ER54458 and 59. Reproduction, translation, publication, use and disposal, in whole or in part, by or for the United States government is permitted. 


\title{
Fluctuation Driven Transport and Stationary Profiles
}

\author{
J. Kesner, ${ }^{1, \text { a) }}$ D.T. Garnier, ${ }^{2}$ and M.E. Mauel ${ }^{2}$ \\ 1) Plasma Science and Fusion Center, Massachusetts Institute of Technology \\ 2) Department of Applied Physics, Columbia University
}

(Dated: 8 June 2011)

Transport equations for particles and energy can be derived when the fluctuations conserve adiabatic invariants. The transport equations determine both stationary density and pressure profiles and the direction of the turbulence-driven fluxes which can be inward or outward. An inward turbulent pinch is predicted which creates stationary profiles and reverses direction depending on the density and temperature gradients. The transport fluxes are independent of the underlying drive that leads to plasma turbulence. For low frequency turbulence the formulation remains correct when the collisional time scale is faster than the confinement time scale.

a) Electronic mail: kesner@psfc.mit.edu 
Self-consistent estimates of transport driven by turbulence usually require computerintensive non-linear calculations. However, for broadband low frequency turbulence, a crossfield flow proportional to the fluctuation intensity can be derived following the ergodic hypothesis $^{1}$ (also known as turbulent equipartiton) when the fluctuations conserve constants of motion $^{2-5}$. The resulting transport equations determine stationary density and pressure profiles and the direction of the associated turbulence-driven fluxes. When the wave-particle interaction is independent of pitch angle, the kinetic transport equations become identical to the MHD quasi-linear transport equations ${ }^{6}$. The experimental observation of stationary profiles thus can serve as an indicator that turbulence-driven cross-field transport is broadband and of sufficient level to dominate transport processes.

For plasma confined by a strong magnetic dipole, both pressure driven MHD modes and low frequency "entropy modes" conserve the adiabatic invariants $\mu$ and J with $\mu$ the magnetic moment,$\mu=v_{\perp}^{2} / 2 B$, and $J$ the longitudinal invariant, $J=\oint v_{\|} d \ell$. In the Levitated Dipole Experiment $(\mathrm{LDX})^{8}$ a superconducting magnet is levitated in a large vacuum chamber so as to avoid plasma losses parallel to the magnetic field lines. When the dipole coil is levitated (as opposed to being mechanically supported) plasma confinement is observed to improve markedly ${ }^{9,10}$, centrally-peaked density profiles result, and we observe a strong inward particle pinch that is consistent with the measured fluctuation level ${ }^{11,12}$. The observed density profiles are usually close to the stationary profiles as derived from $\mathrm{MHD}^{6}$ or from turbulent equipartiton $n^{7}$. When the magnet is supported rather than levitated, the pinch is not observed. Observations of trapped particles in the earth's magnetosphere show similar inward fluxes driven by the random electric and magnetic fields that result from a variable solar wind ${ }^{13}$.

Consider a turbulent spectrum of fluctuations in a collisionless plasma that satisfies $\Omega_{c j} \gg$ $\omega_{b j} \gg \omega$ with $\Omega_{c j}, \omega_{b j}, \omega$ respectively the cyclotron frequency and bounce frequency for species $j$ and the wave frequency. Under these conditions the adiabatic invariants $\mu$ and $J$ are conserved $^{2}$. The flux function, $\psi$, an approximation for the canonical angular momentum, is conjugate with the toroidal angle $\phi$. In the absence of fluctuations $\psi$ is conserved. The toroidal curvature drift is $\omega_{d}$ and in the presence of fluctuations for which $\omega \sim \omega_{d}, \psi$ is no longer conserved. Under this circumstance we can write a Lagrangian collisionless kinetic 
equation for $f=f(\mu, J, \psi, t)^{13,17-19}$ (ignoring density and heating sources) as follows:

$$
\frac{\partial f}{\partial t}=\left.\left.\frac{\partial}{\partial \psi}\right|_{J, \mu} D^{\psi} \frac{\partial f}{\partial \psi}\right|_{J, \mu}
$$

Where we have assumed that the turbulent transport dynamics can be parametrized by a turbulent diffusion coefficient, $D^{\psi}(\mu, J, \psi)$ acting in flux space ${ }^{7,20}, D^{\psi}=2 \tau_{c} R^{2} E_{\phi}^{2}$, and $\tau_{c}$ is the correlation time of the broad-band drift-resonant fluctuations. The particle flux implied by Eq. (1) is

$$
\Gamma=-\left.\iint d \mu d J D^{\psi} \frac{\partial f}{\partial \psi}\right|_{J, \mu} .
$$

In a tokamak the diffusion coefficient $D^{\psi}$ is a function of pitch angle and "passing" particles have a weak response to the fluctuations. Furthermore, trapped particle modes tend to localize on the outside of the torus and therefore deeply trapped particles are more strongly diffused than shallowly trapped particles. When the diffusion coefficient depends on pitch angle, the fluxes will depend on the details of this dependence ${ }^{7}$.

In a dipole there is no rotational transform and therefore no toroidally streaming "passing" particles. Furthermore it has been shown that both $\mathrm{MHD}^{22}$ and entropy modes are flute-like ${ }^{23}$. Noting that $\int d \mu d J=\oint d \ell / B \int d^{3} v$ we can integrate Eq. (1) over $\mu$ and $J$ taking $D^{\psi}=D$, independent of $\psi$ for simplicity, and we obtain:

$$
\frac{\partial N}{\partial t}=D \frac{\partial^{2} N}{\partial \psi^{2}}
$$

with $N \equiv n(\psi) U(\psi)$ and $U \equiv d V / d \psi=\oint d \ell / B$ is the magnetic flux-tube specific volume. Thus, in a collisionless plasma, low frequency turbulence will tend to drive $N(\psi)$ towards the stationary state $N=$ constant when one boundary has a zero flux boundary condition.

In the usual limit of a large collision rate compared to the diffusion rate the distribution function, $f$, must be taken as a local Maxwellian, $f_{M}=(m / 2 \pi T(\psi))^{3 / 2} n(\psi) \exp (-\epsilon / T(\psi))$. Following Ref. ${ }^{7}$, converting variables from $(\mu, J)$ to $(\mu, \epsilon)$ using $d J=d \epsilon(\partial J / \partial \epsilon)_{\psi}$ and using $d \epsilon /\left.d \psi\right|_{J}=-\partial J /\left.\partial \psi\right|_{\epsilon} / \partial J /\left.\partial \epsilon\right|_{\psi}$ and

$$
\left.\frac{\partial f}{\partial \psi}\right|_{J, \mu}=\left.\frac{\partial f}{\partial \psi}\right|_{\epsilon, \mu}+\left.\left.\frac{\partial f}{\partial \epsilon}\right|_{\psi, \mu} \frac{\partial \epsilon}{\partial \psi}\right|_{J, \mu}
$$

we can obtain for both the particle and energy density (i.e. pressure) flux:

$$
\left[\begin{array}{c}
\Gamma \\
\Gamma_{S}
\end{array}\right]=-\iint\left[\begin{array}{l}
1 \\
\epsilon
\end{array}\right] D^{\psi}\left(\left.\left.\frac{\partial f_{M}}{\partial \psi}\right|_{\epsilon} \frac{\partial J}{\partial \epsilon}\right|_{\psi}-\left.\left.\frac{\partial f_{M}}{\partial \epsilon}\right|_{\psi} \frac{\partial J}{\partial \psi}\right|_{\epsilon}\right) d \epsilon d \mu
$$


Frequently $D^{\psi}=D^{\psi}(\lambda), \lambda=\mu / \epsilon$ with $\lambda=\mu / \epsilon$ the pitch angle related variable. For a sufficiently broad fluctuation spectra all particles will satisfy $\omega_{d} \approx \omega$ thus satisfying the condition for randomizing the flux invariant ${ }^{21}$. In a dipole the plasma can become unstable to entropy modes ${ }^{24}$, and the plasma response would become independent of pitch angle. As a result $D^{\psi}$ will be independent of $\lambda$ and we can then integrate Eq. (5) to again obtain

$$
\Gamma=-D^{\psi} \frac{\partial(n U)}{\partial \psi}, \quad \Gamma_{S}=-D^{\psi} \frac{\partial\left(p U^{\gamma}\right)}{\partial \psi}
$$

with $\gamma=5 / 3$. In MHD $\gamma$ enters as the adiabatic constant. $\Gamma$ can be written as the sum of a diffusive term $(\propto U \partial n / \partial \psi)$ and a pinch term $(\propto n \partial U / \partial \psi)$.

Thus turbulent fluxes are produced by gradients in $(N=n U)$ and $S=p U^{\gamma}$ and they drive the plasma toward stationary states characterized by constant or linear values of $N$ and $S$ (solutions with a linear $\psi$ dependence give rise to a steady-state density or energy flow which implies that an internal floating coil is either absorbing or emitting). Alternatively we can consider that the temperature $(T \equiv p / n)$ approaches a stationary state, $T U^{\gamma-1} \approx$ constant. Thus we obtain the same stationary state for collisionless plasmas, for semi-collisional and for collisional (MHD) plasmas. It has been shown from a quasi-linear development of $\mathrm{MHD}^{6,14}$ that low frequency fluctuations that satisfy a "random phase approximation" lead to transport that can be described as a diffusion in flux space ${ }^{6,13,14}$ identical to Eqs. (6). In the absense of particle and heating sources, the particle number within a flux tube, $N=\oint n d l / B \equiv n U$, and the entropy density parameter, $S \equiv p U^{\gamma}$, are stationary when they are uniform. Furthermore, in the absence of inflows, turbulence creates particle and heat fluxes that flatten gradients and drive $\partial N / \partial \psi, \partial S / \partial \psi \rightarrow 0$.

Since, in a dipole, $U(\psi) \sim \psi^{-4} \sim R^{4}$, with $R$ the major radius, $U$ increases rapidly with radius, and an initially uniform density will exhibit inwards transport producing a particle "pinch"11,15. Thus, while collisions tend to reduce density gradients, turbulence driven transport in strongly magnetized plasmas tend to reduce the gradients of the flux tube content, $N$, leading to inwardly peaked stationary profiles. Although a particle pinch has been observed in tokamaks ${ }^{16}$, the effect of the particle pinch is much more dominant in a dipole because the dipole's rapid magnetic field falloff.

Eqs. (6) can be combined to show the relationship between the direction of the particle flux and the plasma profiles:

$$
\Gamma=\frac{\Gamma_{S} /\left(T U^{\gamma-1}\right)+n_{e} D^{\psi} \frac{d U}{d \psi}(\gamma-1-\eta)}{(1+\eta)},
$$


with $\eta=d \ln T_{e} / d \ln n_{e}$. For strong internal heating the pressure gradient will drive MHD instability when $\Gamma_{S}=\partial\left(p U^{\gamma}\right) / \partial \psi>0$ and it is expected that the pressure profile will be clamped close to the marginal profile. At maginality $\Gamma_{S} \propto \partial\left(p U^{\gamma}\right) / \partial \psi \approx 0$ and Eq. (7) becomes:

$$
\Gamma=n_{e} D^{\psi} \frac{d U}{d \psi}\left(\frac{\gamma-1-\eta}{1+\eta}\right) .
$$

For $\eta>\gamma-1$ the particle flux is negative and the flux is inwards whereas when $\eta<\gamma-1$ it is outwards. The term $d U / d \psi$ indicates that this is a strong effect for a dipole (in a dipole $\left.|d U / d \psi| \propto R^{5}\right)$ and a relatively weak effect for a tokamak-like confinement device. Thus for peaked temperature and/or a flat density gradient the density flux is expected to be inwards and vice versa. From Ref. ${ }^{24}$ we observe that, in a dipole, a low frequency instability termed the entropy mode is unstable for $\eta$ both above and below $\gamma-1$ with a stable zone around $\eta \approx \gamma-1=2 / 3$ when $\partial\left(p U^{\gamma}\right) / \partial \psi \lesssim 0$. The pressure flux $\left(\Gamma_{S}\right)$ term in Eq. (7) can contribute to the density pinch. For a MHD stable device $\Gamma_{S}<0$ and the pinch is increased (Eq. (7)). Similarly, for a stationary density profile $\Gamma_{N} \propto \partial\left(n_{e} U\right) / \partial \psi \approx 0$ ( equal particles per unit flux), we obtain from Eq. (7):

$$
\Gamma_{S}=p U^{\gamma-1} D^{\psi} \frac{d U}{d \psi}(\eta-(\gamma-1))
$$

indicating an outflow of energy occurs when $\eta>\gamma-1$ and vica versa.

Using a local approximation it has been shown ${ }^{25,26}$ from thermodynamics that,

$$
\operatorname{Sign}\left(-n^{\prime}\right)\left(\hat{Q} / T_{0} \eta+3 / 2 \hat{\Gamma}(\gamma-1-\eta)\right)>0
$$

with $\hat{\Gamma}$ the particle flux, $\hat{\Gamma}=\int\left\langle v_{r} \delta f\right\rangle d^{3} v$ and $\hat{Q}$ the energy flux, $\hat{Q}=1 / 2 \int\left\langle v_{r} m v^{2} \delta f\right\rangle d^{3} v$ (notice $U$ does not appear). Thus, in the absence of thermal transport, thermodynamics requires inward particle transport, (i.e. a pinch, $\hat{\Gamma}<0$ ) when $\eta>\gamma-1$ and vica versa. In the absence of particle transport $(\hat{\Gamma} \approx 0)$ and for $\eta>0$, thermal transport is always outward. Inwards thermal transport in the small $\eta$ limit, as implied by Eq. (9), requires that the turbulent diffusion be externally driven.

Non-linear simulations of stability in a dipole have been carried out in both a dipole and a Z-pinch configuration. These studies show the growth and non-linear saturation of entropy modes ${ }^{27,28}$. These simulations show the presence of a pinch when the entropy mode is linearly unstable. Furthermore the entropy mode is seen to change character at $\eta=2 / 3$ resulting in a change of the sign of the $\operatorname{pinch}^{29}$. In the approach described here we 
only assume the presence of a turbulent spectrum that conserves $\mu$ and $J$ (in the Z-pinch $\left.J=r v_{\|}\right)$. It thus appears that the observed pinch is a generic consequence of low-frequency, broad-band turbulence. The resulting stationary profiles are independent of the intensity of the fluctuations and only depend on the magnetic field structure. On the contrary, tokamaks observe "critical gradient driven turbulence" 30 . In a tokamak, ion temperature gradient (ITG) driven instability relates the temperature profile to the density profile. If the density profile flattens, the ITG turbulence level will rise to constrain the temperature profile to one having marginal stability.

In magnetic geometries not stabilized by good curvature, the density and temperature profiles are determined by the magnetic geometry. Once there is sufficient heating to create stationary profiles, further heating will increase the level of turbulence leading to increased power flows which can off-load the additional heat. The total stored energy and particle content will only change if the changes in the scrape-off-layer flows modify the plasma parameters at the boundary ${ }^{12}$. For a sufficient level of heating, an inward particle pinch is predicted when $\eta>\gamma-1$, which is the usual condition for core heating and edge fueling in laboratory fusion experiments. The special significance of $\eta=\gamma-1$ comes about because this value implies that when $n U$ and $p U^{\gamma}$ are stationary (equal particles and entropy density per unit flux), an exchange of flux tubes will not change the density and temperature profiles. These predictions only require low frequency turbulence which conserves the adiabatic invariants $\mu$ and $J$ and do not depend on the origin of turbulence. If the fluctuations are driven by an external source, such as the random modulations of the solar wind pressure exerted on the Earth's magnetosphere, then both the density and pressure can pinch $^{21}$ and the density and pressure become centrally peaked with $\eta \sim \gamma-1$.

In the quasilinear approach a turbulence-driven evolution of the background variables is obtained by assuming that cross-mode terms average out (the "random phase" approximation) while the growing modes act as a diffusive term on the background profiles. The diffusive modification of the background gradients will reduce the growth rates of the unstable modes (the quasilinear effect) but the unstable modes may also be damped by non-linear coupling to damped modes which was seen to be the dominant effect leading to saturation of the growing modes in the MHD limit ${ }^{6,14}$. Kobayashi et al. also show that the turbulencedriven pinch that derives from the entropy mode can be formulated from quasilinear theory ${ }^{29}$ and again the amplitude of the turbulent-driven diffusion requires nonlinear simulations. 
The recognition that, in the small gyro-radius limit, the motion of charged particles in a dipole field can be defined by the conservation of $\mu, J$ and $\psi$ lead to the formulation of Eq. 1 when rthe $\psi$ invariant is broken. This formulation is shown to produce a diffusion driven pinch which is directed so as to lead to stationary profiles of density and temperature.

\section{Acknowledgements}

This work was supported by the US DoE Office of Fusion Energy Sciences with Grants DE-FG02-98ER54458 and DE-FG02-98ER54459.

\section{REFERENCES}

${ }^{1}$ J. von Neumann, Proc. Nat. Acad. Sci. 18, 263 (1932).

${ }^{2}$ H.P. Warren, A. Bhattacharjee and M.E. Mauel, Geophys. Research Lett. 19, 941 (1992).

${ }^{3}$ M.B. Isichenko, N.V. Pervisshvili, Phys. Plasmas 2, 3650 (1995).

${ }^{4}$ V.V. Yankov, JETP Lett. 60171 (1994).

${ }^{5}$ H.P. Warren and M.E. Mauel, Phys. Plasmas 2, 4185 (1995).

${ }^{6}$ A. Kouznetsov, J.P. Freidberg, J. Kesner, Phys. Plasmas 14, 102501-1-13 (2007).

${ }^{7}$ D. Baker and M.N. Rosenbluth, Phys. Plasmas 5, 2936 (1998).

${ }^{8}$ D.T. Garnier, A.K. Hansen, J. Kesner, M.E. Mauel, P.C. Michael et al., Fusion Eng. and Design 81, $2371(2006)$.

${ }^{9}$ D.T. Garnier et al., Phys Plasmas 13, 056111 (2006).

${ }^{10}$ D.T. Garnier, et al., Nuc. Fusion 49, 055023 (2009).

${ }^{11}$ A.C. Boxer et al., Nature Phys., doi:10.1038/nphys1510 (2010).

${ }^{12}$ J. Kesner, M. S. Davis, J.L. Ellsworth, D. T. Garnier, J. Kahn, M. E. Mauel, P. Michael, B. Wilson and P. P. Woskov, Plasma Phys. Contr. Fusion 52, 124036 (2010).

${ }^{13}$ T. Birmingham, J. Geophys. Res. 74, 2169 (1969).

${ }^{14}$ V. Pastukhov and N. Chudin, Plasma Physics Reports 27, 963-977 (2001).

${ }^{15}$ A. Hasegawa, Phys. Scripta, T116, 72 (2005).

${ }^{16}$ D. Baker, Phys. Plasmas 9, 2675 (2002).

${ }^{17}$ I.B. Bernstein and K. Molvig, Phys. Plasmas 26 , 1488 (1983).

${ }^{18}$ M.B. Isichenko, A.V. Gruzinov, P.H. Diamond, Phys. Rev. Lett. 74, 4436 (1995). 
${ }^{19}$ M.B. Isichenko, A.V. Gruzinov, P.H. Diamond and P.N. Yushmanov, Phys. Plasmas, 3, 1916 (1996).

${ }^{20}$ X. Garbet et al., Phys. Rev. Lett. 91, 035001 (2003).

${ }^{21}$ A. Hasegawa, Comm. Pl. Phys \& Cont. Fus. 1, 147 (1987).

${ }^{22}$ D.T. Garnier, J. Kesner, M.E. Mauel, Phys. Plasmas 6, 3431 (1999).

${ }^{23}$ J. Kesner, and R. Hastie, Phys. Plasmas 9, 395 (2002).

${ }^{24}$ J. Kesner, Phys. Plasmas 7, 3837 (2000).

${ }^{25}$ K. Hallatschek, Phys. Rev. Lett. 93, 125001-1 (2004).

${ }^{26}$ X. Garbet et al., Phys. Plasmas 12, 082511 (2005).

${ }^{27}$ P. Ricci, B. Rogers, and W. Dorland, Phys. Rev. Lett. 97, 245001 (2006).

${ }^{28}$ S. Kobayashi, B. Rogers, W. Dorland, Phys. Rev. Lett. 103, 055003 (2009).

${ }^{29}$ S. Kobayashi, B. Rogers, W. Dorland, Phys. Rev. Lett. 105, 235004 (2010).

${ }^{30}$ B. Coppi, M.N. Rosenbluth and R.Z. Sagdeev, Phys. Fluids 10, 582 (1967). 